\title{
Nuclear Neutrino Spectra in Late Stellar Evolution
}

\author{
G. Wendell Misch ${ }^{1,2, *}$, Yang Sun ${ }^{1,2,3, * *}$, and George Fuller ${ }^{4, * * *}$ \\ ${ }^{1}$ School of Physics and Astronomy, Shanghai Jiao Tong University, Shanghai 200240, China \\ ${ }^{2}$ Collaborative Innovation Center of IFSA, Shanghai Jiao Tong University, Shanghai 200240, China \\ ${ }^{3}$ Institute of Modern Physics, Chinese Academy of Sciences, Lanzhou 730000, China \\ ${ }^{4}$ Department of Physics, University of California, San Diego La Jolla, CA 92093, USA
}

\begin{abstract}
Neutrinos are the principle carriers of energy in massive stars, beginning from core carbon burning and continuing through core collapse and after the core bounce. In fact, it may be possible to detect neutrinos from nearby pre-supernova stars. Therefore, it is of great interest to understand the neutrino energy spectra from these stars. Leading up to core collapse, beginning around core silicon burning, nuclei become dominant producers of neutrinos, particularly at high neutrino energy, so a systematic study of nuclear neutrino spectra is desirable. We have done such a study, and we present our $s d$-shell model calculations of nuclear neutrino energy spectra for nuclei in the mass number range $A=21-35$. Our study includes neutrinos produced by charged lepton capture, charged lepton emission, and neutral current nuclear deexcitation. Previous authors have tabulated the rates of charged current nuclear weak interactions in astrophysical conditions, but the present work expands on this not only by providing neutrino energy spectra, but also by including the heretofore untabulated neutral current de-excitation neutrino pairs.
\end{abstract}

\section{Introduction}

Neutrinos from nearby $(<1 \mathrm{kpc})$ presupernova stars, of which there are several candidates, may soon be detectable with Earth-based neutrino detection experiments [1-3]. Therefore, there has been recent work to produce realistic neutrino spectra from presupernova cores. In the final phases before collapse, when the star begins to fuse silicon into iron, nuclei become the dominant sources of neutrinos, particularly at high energy $[4,5]$.

Reference [5] presented expected presupernova neutrino spectra and detector signals from simulation results. However, they used the technique of reference [6] to compute the nuclear component of the spectra from published nuclear weak rate tables. This method consists of treating each nucleus at each temperature-density point as having a single transition from parent to daughter with an effective Q-value chosen to reproduce the published average neutrino energy. However, reference [7] showed that nuclear structure must be properly considered to obtain accurate nuclear neutrino spectra; electron/positron captures and decays can in principle proceed from any state in the parent nucleus to any energetically-allowed state in the daughter nucleus. These two approaches are shown schematically in figure 1 .

\footnotetext{
*e-mail: wendell.misch@gmail.com

**e-mail: sunyang@sjtu.edu.cn

***e-mail: gfuller@ucsd.edu
} 

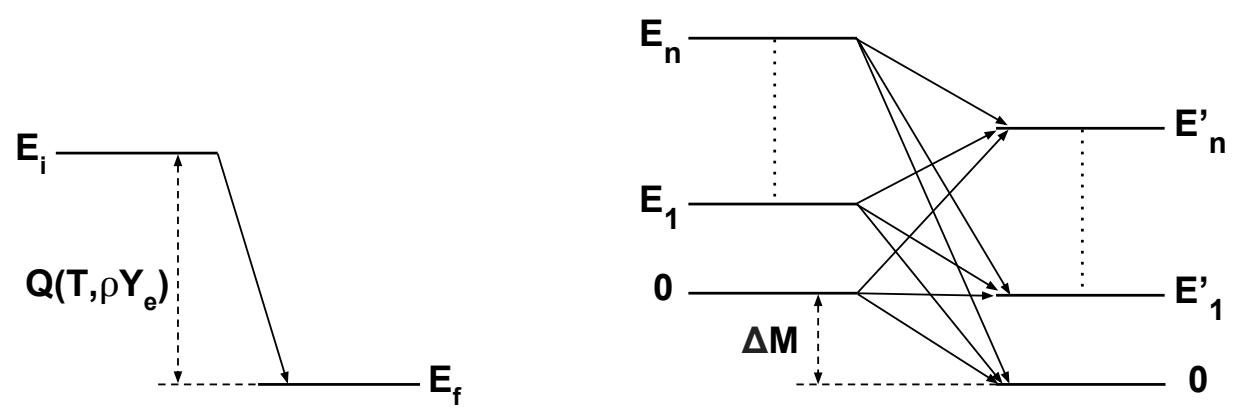

Figure 1. Two approaches to computing the nuclear contribution to neutrino spectra. Left: Single effective Q-value method. For each nucleus at each temperature and density, assume a single effective transition from the parent to the daughter with a Q-value chosen to reproduce the average neutrino energy in published rate tables. Right: Full structure method. Consider all transitions between thermally populated initial parent states and final daughter states.

We also show in section 2 that neutral current nuclear de-excitation, wherein an excited nucleus relaxes by emitting a virtual $Z^{0}$ boson that decays to a $v-\bar{v}$ pair, is likely a dominant source of high-energy antineutrinos late in silicon burning and in early collapse, but it is generally not included in stellar models.

\section{Results}

Because nuclei are the dominant source of detectable neutrinos and because nuclear structure is an important component of producing realistic spectra, we have produced a catalog of neutrino spectra for $70 s d$-shell nuclei on the Fuller-Fowler-Newman [8] temperature-density grid. We present here a small sample of spectra from our tables.

Figure 2 shows neutrino spectra from electron capture and positron decay of ${ }^{27} \mathrm{Si}$ at a temperature and density appropriate for a silicon-burning stellar core. Structure effects are evident as features in the spectra, particularly near $\sim 5 \mathrm{MeV}$. The features are due to transitions proceeding through different parent and/or daughter nuclear states.
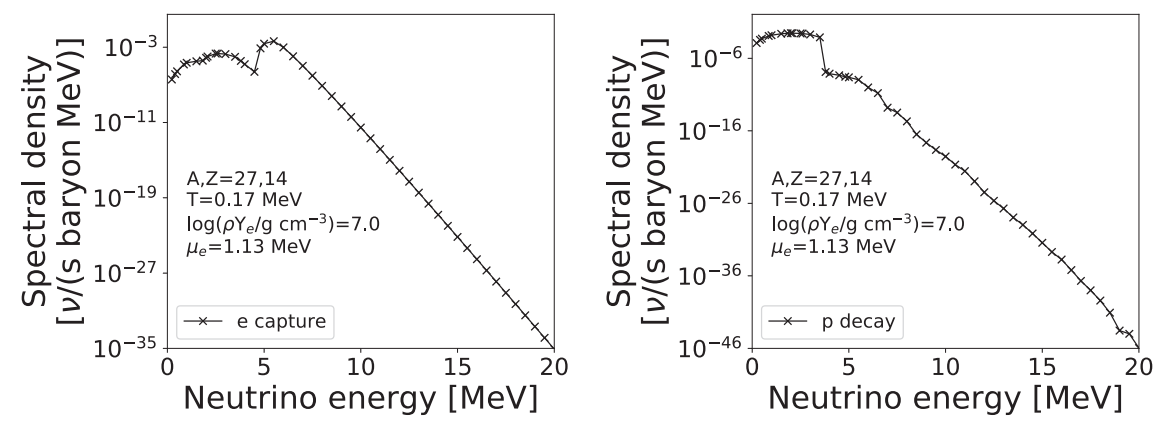

Figure 2. Neutrino spectra from electron capture (left) and positron decay (right) in ${ }^{27} \mathrm{Si}$ at temperature $T=0.17 \mathrm{MeV}$ and $\rho Y_{e}=10^{7} \mathrm{~g} / \mathrm{cm}^{3}$. Nuclear structure manifests as features in the spectra. 

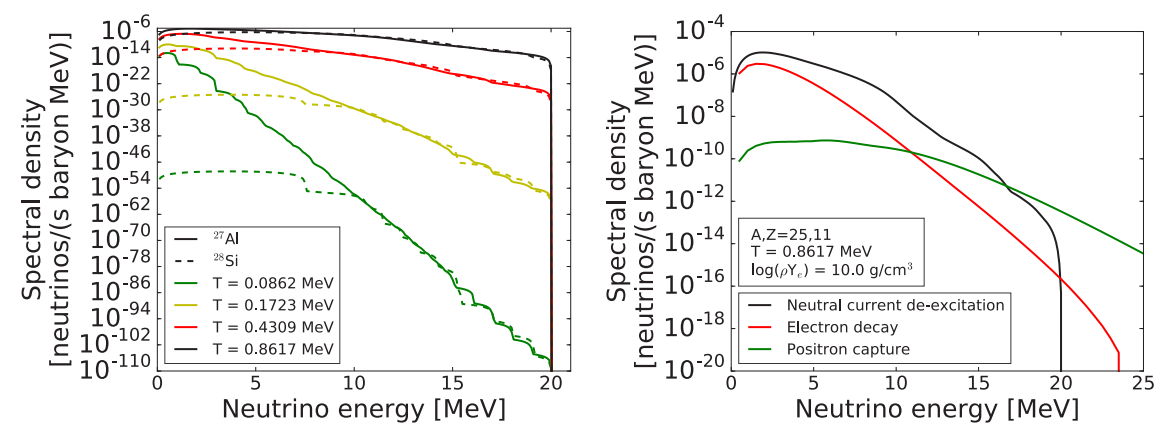

Figure 3. Left panel: Neutral current de-excitation neutrino spectra for ${ }^{27} \mathrm{Al}$ (solid) and ${ }^{28} \mathrm{Si}$ (dashed) at various temperatures leading up to collapse. Right panel: Antineutrino spectra from ${ }^{25} \mathrm{Na}$ in early collapse conditions. Neutral current de-excitation is extremely sensitive to temperature, and in early collapse conditions, it is a dominant source of high energy antineutrinos.

Figure 3 shows neutrino spectra from de-excitation of ${ }^{27} \mathrm{Al}$ and ${ }^{28} \mathrm{Si}$ at a wide range of temperatures along with a comparison of antineutrino spectra from three processes in ${ }^{25} \mathrm{Na}$ under early collapse conditions. Neutral current de-excitation is incredibly sensitive to temperature, increasing by orders of magnitude with a factor of 2 increase in temperature. Because no charged leptons are involved, this process is independent of density.

${ }^{25} \mathrm{Na}$ will not be abundant in early collapse, but it has the correct electron-to-baryon ratio $Y_{e}$, so it serves as a proxy for more realistic nuclei. In these conditions, neutral current de-excitation antineutrinos dominate both positron capture and electron decay up to at least $17 \mathrm{MeV}$ neutrino energy. Because we do not include initial states above $\sim 20 \mathrm{MeV}$ in our calculations, the neutral current spectra cut off sharply at $20 \mathrm{MeV}$. Therefore, it may be that this process continues to dominate at even higher neutrino energies. Regardless, it is clear that a realistic estimate of pre-supernova neutrino spectra must include neutral current nuclear de-excitation.

The techniques used to produce these spectra are detailed in reference [9]. Our complete nuclear neutrino spectra tables are available for download and use at http://www.jinaweb.org/ $\mathrm{html} / \mathrm{mischnuspectra.html.}$

\section{References}

[1] A. Odrzywolek, M. Misiaszek, M. Kutschera, Acta Physica Polonica B 35, 1981 (2004)

[2] A. Odrzywolek, M. Misiaszek, M. Kutschera, Astroparticle Physics 21, 303 (2004)

[3] K. Asakura, A. Gando, Y. Gando, T. Hachiya, S. Hayashida, H. Ikeda, K. Inoue, K. Ishidoshiro, T. Ishikawa, S. Ishio et al., ApJ 818, 91 (2016)

[4] A. Odrzywolek, A. Heger, Acta Physica Polonica B 41, 1611 (2010)

[5] K.M. Patton, C. Lunardini, R.J. Farmer, The Astrophysical Journal 840, 2 (2017)

[6] K. Langanke, G. Martínez-Pinedo, J.M. Sampaio, Phys. Rev. C 64, 055801 (2001)

[7] G.W. Misch, G.M. Fuller, Phys. Rev. C 94, 055808 (2016)

[8] G.M. Fuller, W.A. Fowler, M.J. Newman, ApJ 252, 715 (1982)

[9] G.W. Misch, Y. Sun, G.M. Fuller, ApJ 852, 43 (2018) 